\title{
Number of negative lymph nodes is associated with disease-free survival in patients with breast cancer
}

San-Gang Wu ${ }^{1+}$, Jia-Yuan Sun ${ }^{2 \dagger}$, Juan Zhou ${ }^{3+}$, Feng-Yan Li ${ }^{2}$, Qin Lin ${ }^{1}$, Huan-Xin Lin ${ }^{2}$, Xun-Xing Guan ${ }^{2}$ and Zhen-Yu He $\mathrm{H}^{2^{*}}$

\begin{abstract}
Background: The aim of this study was to evaluate the prognostic value of the number of negative lymph nodes (NLNs) in breast cancer patients after mastectomy.

Methods: 2,455 breast cancer patients who received a mastectomy between January 1998 and December 2007 were retrospectively reviewed. The prognostic impact of the number of NLNs with respect to disease-free survival (DFS) was analyzed.

Results: The median follow-up time was 62.0 months, and the 5-year and 10-year DFS was $87.1 \%$ and $74.3 \%$, respectively. The DFS of patients with $>10$ NLNs was significantly higher than that of patents with $\leq 10$ NLNs, and the 5 -year DFS rates were $87.5 \%$ and $69.5 \%$, respectively $(P<0.001)$. Univariate Cox analysis showed that the NLN count (continuous variable) was a prognostic factor of DFS (hazard ratio $[\mathrm{HR}]=0.913,95 \%$ confidence interval [Cl]: 0.896-0.930, $P<0.001$ ). In multivariate Cox analysis, patients with a higher number of NLNs had a better DFS ( $H R=0.977,95 \% \mathrm{Cl}: 0.958-0.997, P=0.022)$. Subgroup analysis showed that the NLN count had a prognostic value in patients at different $\mathrm{pT}$ stages and pN positive patients (log-rank $P<0.001$ ). However, it had no prognostic value in pNO patients (log-rank $P=0.684)$.
\end{abstract}

Conclusions: The number of NLNs is an independent prognostic factor of DFS in breast cancer patients after mastectomy, and patients with a higher number of NLNs have a better DFS.

Keywords: Breast cancer, Mastectomy, Negative lymph nodes, Prognosis, Disease-free survival

\section{Background}

Though the survival rates of patients receiving sentinel lymph node biopsy and of patients receiving axillary lymph node dissection are similar in a certain specific populations with breast cancer [1,2], and sentinel lymph node biopsy can decrease postoperative arm lymphedema $[3,4]$, the axillary lymph node status is still one of the most important prognostic indicators of breast cancer patients and is useful for guiding treatment. Moreover, it is also important in the Union for International Cancer Control/American Joint Committee on Cancer

\footnotetext{
* Correspondence: hezhy@sysucc.org.cn

${ }^{\dagger}$ Equal contributors

2Department of Radiation Oncology, Sun Yat-sen University Cancer Center, State Key Laboratory of Oncology in South China, Collaborative Innovation Center of Cancer Medicine, Guangzhou 510060, People's Republic of China Full list of author information is available at the end of the article
}

(UICC/AJCC) tumor, node, metastasis (TNM) staging system for breast cancer.

Axillary lymph node dissection is an important method for determining the axillary lymph node status in breast cancer patients. In theory, the survival of breast cancer patients is improved by removing more axillary lymph nodes. However, the prognostic value of the number of axillary lymph nodes removed is controversial [5-7]. Because both positive and negative lymph nodes are removed, it is difficult to accurately determine the proper number of lymph nodes that should be removed.

The number of negative lymph nodes (NLNs) removed is obtained by subtracting the number of positive lymph nodes from the total number of removed lymph nodes. Because removing more NLNs may reduce the possibility of occult lesions and thus improve the prognosis, the 
number of NLNs removed may be an indicator of the degree of the appropriateness of axillary lymph node dissection. The prognostic value of the number of NLNs removed in esophageal cancer, colorectal cancer, and cervical cancer has been proven [8-11]. However, there have been few studies on its prognostic value of the number of NLNs removed for breast cancer $[12,13]$. The purpose of this study was to determine the prognostic value of the number of NLNs with respect to diseasefree survival (DFS) of breast cancer patients after mastectomy.

\section{Methods \\ Patients}

The records of breast cancer patients treated at Sun YatSen University Cancer Center between January 1998 and December 2007 were retrospectively reviewed. The inclusion criteria were: 1) Females who had histologically confirmed unilateral invasive breast cancer; 2) Underwent mastectomy together with axillary lymph node dissection and the number of removed axillary lymph nodes was more than $10 ; 3$ ) The tumor was completely removed and the margins were negative; 4) No neoadjuvant chemotherapy was administered before surgery and postoperative treatments including chemotherapy, radiotherapy, and endocrine therapy were performed based on the tumor stage and hormone receptor status. The study was approved by the ethics committee of Sun YatSen University Cancer Center. All patients provided written consent for storage of their information in the hospital database, and for use of this information for research purposes.

\section{Clinicopathologic factors and lymph node status}

The risk of recurrence was evaluated according to the clinicopathological characteristics and immunohistochemical factors which included age, menopause status, pT stage, pN stage, and estrogen receptor (ER), progesterone receptor (PR), and human epithelial growth factor receptor family 2 (Her2) status. ER and PR positive was defined as more than $1 \%$ positive cells on immunohistochemical analysis. Her2-positivity was defined as a 3+ immunohistochemical result or a $2+$ immunohistochemical result confirmed by fluorescent in situ hybridization (FISH). pT stage and pN stage were consistent with the UICC/AJCC TNM classification ( $7^{\text {th }}$ Edition), and $\mathrm{pN}$ stages were defined as follows: pNO, no regional lymph node metastasis identified histologically; $\mathrm{pN} 1$, metastasis in 1-3 lymph nodes; pN2, metastasis in 4-9 lymph nodes; pN3, metastasis in $\geq 10$ lymph nodes. The number of removed NLNs was defined as the number of positive lymph nodes subtracted from the total number of removed lymph nodes.

\section{Histopathological examination of resected lymph nodes}

All resected specimens were submitted for pathologic examination. Pathologists examined all slides to evaluate the depth of the primary tumors and node involvement, which were separately labeled by the surgeons in a routine manner. One section from each lymph node was analyzed after hematoxylin and eosin (H\&E) staining. Lymph nodes that were examined included those that were embedded in the en bloc specimen and not labeled by surgeons, but were identified by the pathologists. The lymph node number was counted on low-power field microscopy. The total number of resected lymph nodes was the sum of the lymph nodes removed form the axilla. The number of metastatic lymph nodes, and the number of removed nodes was determined.

\section{Follow-up and survival endpoints}

Follow up was performed 3-6 months after surgery by hospital visit, telephone, or mail correspondence. Because all patients in the present study received adjuvant treatment according to the stage and hormone receptor status, the endpoint was DFS. For patients with recurrence, survival time was determined from the date of surgery to the date of locoregional recurrence and/or distant metastasis.

\section{Statistical analysis}

The $\chi^{2}$ and Fisher's exact tests were used to analyze the differences between qualitative data. Recognizing that the total number of NLNs removed may be subjected to incomplete counting or natural interindividual variation in nodal distribution, the variable was examined as a categorical variable based on quartiles. Calculation of survival rates were plotted by the Kaplan-Meier method, and compared using the log-rank test. Univariate and multivariate Cox regression model analyses were performed. All analyses were performed using the SPSS statistical software package version 16.0 (IBM Corporation, Armonk, NY, USA). A value of $P<0.05$ was considered statistically significant.

\section{Results}

\section{Clinicopathological characteristics and lymph node} dissection data

A total of 2,455 patients were included in the analysis, and the clinical features are shown in Table 1. The median number of removed lymph nodes was $15\left(25^{\text {th }}\right.$ percentile $=12,75^{\text {th }}$ percentile $=18$; range, $\left.10-73\right), 1,263$ patients were node negative (51.4\%), and 1,192 patients were node positive $(48.6 \%)$. Of the patients, 769, 207 and 216 were $\mathrm{pN} 1, \mathrm{pN} 2$ and $\mathrm{pN} 3$, respectively.

The median number of NLNs removed was 13 (25th percentile $=11,75$ th percentile $=16$; range, $0-40$ ). Examination of the number of NLNs removed as a categorical 
Table 1 Correlation between the number of negative lymph nodes removed and clinicopathologic factors

\begin{tabular}{|c|c|c|c|c|c|c|}
\hline Characteristic & & \multicolumn{4}{|c|}{ Number of negative lymph nodes } & $P$ value \\
\hline \multicolumn{7}{|l|}{ Age } \\
\hline$<35$ & 234 & $68(11.2)$ & $51(7.2)$ & $51(9.2)$ & $64(10.9)$ & \multirow[t]{2}{*}{0.054} \\
\hline$\geq 35$ & 2221 & $539(88.8)$ & $655(92.8)$ & $503(90.8)$ & $524(89.1)$ & \\
\hline \multicolumn{7}{|l|}{ Menopause } \\
\hline Premenopause & 1641 & $402(66.2)$ & $460(65.2)$ & 354 (63.9) & 425 (72.3) & \multirow[t]{2}{*}{$0.012^{*}$} \\
\hline Postmenopause & 814 & $205(33.8)$ & $246(34.8)$ & $200(36.1)$ & $163(27.7)$ & \\
\hline \multicolumn{7}{|l|}{ pT stage } \\
\hline $\mathrm{T} 1$ & 812 & $159(26.2)$ & $259(36.7)$ & $190(34.3)$ & $204(34.7)$ & \multirow[t]{4}{*}{$<0.001^{*}$} \\
\hline $\mathrm{T} 2$ & 1428 & $352(58.0)$ & $398(53.4)$ & $323(58.3)$ & $355(60.4)$ & \\
\hline T3 & 155 & $71(11.7)$ & $41(5.8)$ & $25(4.5)$ & $18(3.1)$ & \\
\hline T4 & 60 & $25(4.1)$ & $8(1.1)$ & $16(2.9)$ & $11(1.8)$ & \\
\hline \multicolumn{7}{|l|}{ pN stage } \\
\hline No & 1263 & $120(19.8)$ & $430(60.9)$ & $342(61.7)$ & $371(63.1)$ & \multirow[t]{4}{*}{$<0.001^{*}$} \\
\hline N1 & 769 & $185(30.5)$ & $220(31.2)$ & 174 (31.4) & $190(32.3)$ & \\
\hline N2 & 207 & $115(18.9)$ & $44(6.2)$ & $30(5.4)$ & $18(3.1)$ & \\
\hline N3 & 216 & $187(30.8)$ & $12(1.7)$ & $8(1.5)$ & $9(1.5)$ & \\
\hline \multicolumn{7}{|l|}{ ER } \\
\hline Negative & 953 & $245(40.4)$ & $252(35.7)$ & 219 (39.5) & $237(40.3)$ & \multirow[t]{3}{*}{0.304} \\
\hline Positive & 1292 & $321(52.9)$ & $384(54.3)$ & $287(51.8)$ & $300(51.0)$ & \\
\hline Unknown & 210 & $41(6.7)$ & 70 (10.0) & $48(8.7)$ & $51(8.7)$ & \\
\hline \multicolumn{7}{|l|}{$P R$} \\
\hline Negative & 824 & 207 (34.1) & $223(31.5)$ & $198(35.7)$ & $196(33.3)$ & \multirow[t]{3}{*}{0.398} \\
\hline Positive & 1421 & $359(59.1)$ & $413(58.5)$ & $308(55.6)$ & $341(58.0)$ & \\
\hline Unknown & 210 & $41(6.8)$ & $70(10.0)$ & $48(8.7)$ & $51(8.7)$ & \\
\hline \multicolumn{7}{|l|}{ Her-2 } \\
\hline Negative & 1377 & $329(54.2)$ & $395(56.0)$ & $307(55.4)$ & $346(58.8)$ & \multirow[t]{3}{*}{0.460} \\
\hline Positive & 666 & $168(27.7)$ & $183(25.9)$ & $157(28.3)$ & $158(26.9)$ & \\
\hline Unknown & 412 & $110(18.1)$ & $128(18.1)$ & $90(16.3)$ & $84(14.3)$ & \\
\hline
\end{tabular}

Data are presented as number (percentage).

ER, estrogen receptor; PR, progesterone receptor; Her-2, human epidermal growth factor receptor-2.

${ }^{*} P<0.05$ indicates a significant difference.

variable based on quartiles showed the number of patients with 0-10 NLNs removed (group 1) was 607, the number with 11-13 NLNs removed (group 2) was 706, the number with 14-16 NLNs removed (group 3) was 554, and the number with 17-40 NLNs removed (group 4) was 588.

The NLN count was associated with menopause status, pT stage, and $\mathrm{pN}$ stage $(P<0.05)$. The NLN count was not associated with age or ER, PR, and Her-2 status (all, $P>0.05$ ) (Table 1).

\section{Relationship between the number of NLNs removed and DFS}

The median follow-up time was 62.0 months (range, 6 to 158 months), and 477 patients had local recurrence and/or distant metastasis. The 5- and 10-year DFS rates were $81.7 \%$ and $74.3 \%$, respectively. The number of NLNs removed had a significant impact on DFS, the 5year DFS in group 1, group 2, group 3, and group 4 were $69.5 \%, 86.0 \%, 83.3 \%$, and $87.6 \%$, respectively. The $10-$ year DFS in group 1, group 2, group 3, and group 4 were $61.8 \%, 76.8 \%, 78.5 \%$, and $81.7 \%$, respectively $(P<0.001)$ (Figure 1A). However, intersection and overlapping were observed between the survival curves of patients in group 2, 3 and 4; therefore, we combined these 3 groups. The analysis with these groups combined showed that the 5- and 10-year DFS rates were $85.7 \%$ and $78.5 \%$ in patients with $>10$ NLNs removed, which were significantly higher than $69.5 \%$ and $61.8 \%$ of patients with $\leq 10$ NLNs removed $(P<0.001)$ (Figure $1 \mathrm{~B})$. 

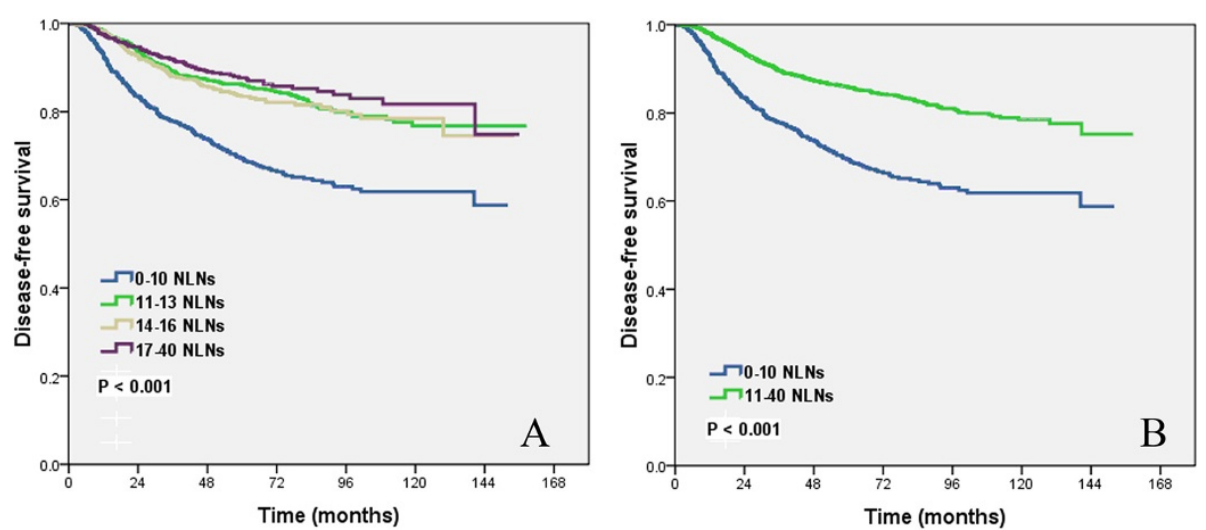

Figure 1 Impact of the number of negative lymph nodes on disease-free survival for 4 categories of patients (A), and patients with group 1 vs. group 2-4 (B).

Table 2 Univariate and multivariate analyses of disease-free survival

\begin{tabular}{|c|c|c|c|c|c|c|}
\hline \multirow[t]{2}{*}{ Characteristic } & \multicolumn{3}{|c|}{ Univariate } & \multicolumn{3}{|c|}{$\underline{\text { Multivariate }}$} \\
\hline & HR & $95 \% \mathrm{Cl}$ & $P$ value & $\mathrm{HR}$ & $95 \% \mathrm{Cl}$ & $P$ value \\
\hline \multicolumn{7}{|l|}{ Age } \\
\hline$<35$ & 1 & & & 1 & & \\
\hline$\geq 35$ & 0.618 & $0.476-0.803$ & $<0.001^{*}$ & 0.679 & $0.522-0.883$ & $0.004^{*}$ \\
\hline \multicolumn{7}{|l|}{ Menopause } \\
\hline Premenopause & 1 & & & - & & \\
\hline Postmenopause & 0.995 & $0.822-1.205$ & 0.960 & - & & \\
\hline \multicolumn{7}{|l|}{ pT stage } \\
\hline $\mathrm{T} 1$ & 1 & & & 1 & & \\
\hline $\mathrm{T} 2$ & 1.699 & $1.361-2.120$ & $<0.001^{*}$ & 1.361 & $1.088-1.704$ & $0.007^{*}$ \\
\hline T3 & 2.844 & 2.036-3.972 & $<0.001^{*}$ & 1.585 & $1.116-2.252$ & $0.010^{*}$ \\
\hline T4 & 3.150 & $1.933-5.134$ & $<0.001^{*}$ & 1.798 & $1.091-2.963$ & $0.021^{*}$ \\
\hline \multicolumn{7}{|l|}{ pN stage } \\
\hline No & 1 & & & 1 & & \\
\hline $\mathrm{N} 1$ & 2.187 & $1.746-2.740$ & $<0.001^{*}$ & 2.083 & $1.658-2.616$ & $<0.001^{*}$ \\
\hline N2 & 3.139 & $2.322-4.244$ & $<0.001^{*}$ & 2.506 & $1.817-3.456$ & $<0.001^{*}$ \\
\hline N3 & 6.725 & $5.236-8.638$ & $<0.001^{*}$ & 4.575 & $3.315-6.313$ & $<0.001^{*}$ \\
\hline \multicolumn{7}{|l|}{ ER } \\
\hline Negative & 1 & & & 1 & & \\
\hline Positive & 0.586 & $0.504-0.682$ & $<0.001^{*}$ & 0.720 & $0.582-0.892$ & $0.003^{*}$ \\
\hline \multicolumn{7}{|l|}{ PR } \\
\hline Negative & 1 & & & 1 & & \\
\hline Positive & 0.620 & $0.533-0.722$ & $<0.001^{*}$ & 0.790 & $0.636-0.980$ & $0.032^{*}$ \\
\hline \multicolumn{7}{|l|}{ Her2 } \\
\hline Negative & 1 & & & - & & \\
\hline Positive & 0.977 & $0.871-1.096$ & 0.693 & - & & \\
\hline Number of NLNs (continuous variable) & 0.913 & $0.896-0.930$ & $<0.001^{*}$ & 0.977 & $0.958-0.997$ & $0.022^{*}$ \\
\hline
\end{tabular}

DFS, disease-free survival; $\mathrm{HR}$, hazard ratio; $\mathrm{Cl}$, confidence interval; ER estrogen receptor; PR, progesterone receptor; Her-2, human epidermal growth factor receptor-2; NLNs, negative lymph nodes.

${ }^{*} P<0.05$ indicates a significant difference. 


\section{Analysis of prognostic factors}

Univariate analysis showed that age, $\mathrm{pT}$ stage, $\mathrm{pN}$ stage, ER status, PR status, and the number of NLNs removed (continuous variables) were prognostic factors affecting DFS (all, $P<0.001$ ) (Table 2).

Multivariate analysis showed that the number of NLNs removed was an independent prognostic factor of DFS; patients with a higher number of NLNs had a better DFS (hazard ratio $[\mathrm{HR}]=0.977,95 \%$ confidence interval [CI]: 0.958-0.997, $P=0.022$ ). In addition, age, pT stage, $\mathrm{pN}$ stage, ER status, and PR status were also independent risk factors of DFS (all, $P<0.05)$ (Table 2).

\section{Impact of the number of NLNs removed on DFS by $\mathrm{pT}$ stage}

Subgroup analysis of the impact of the number of NLNs removed on DFS by different $\mathrm{pT}$ stage showed that patients with a higher number of NLNs removed at all pT stages had better DFS (log-rank $P<0.001$ for pT1, $P<$ 0.001 for $\mathrm{pT} 2, P<0.001$ for $\mathrm{pT} 3$, and $P<0.001$ for $\mathrm{pT} 4$ ) (Figure 2A-D).

\section{Impact of the number of NLNs removed on DFS by pN stage}

Subgroup analysis of the impact of the number of NLNs removed on DFS by different $\mathrm{pN}$ stage showed that the NLN count removed had no impact on DFS in pNO patients (log-rank $P=0.684$ ). However, a higher number of NLNs removed indicated better DFS in pN-positive patients (log-rank $P<0.001$ ) (Figure 3A,B).

\section{Discussion}

In the present study, we investigated the impact of the number of NLNs removed after mastectomy in breast cancer patients and found that the number of NLNs removed was an independent prognostic factor of DFS. Patients with a higher number of NLNs removed had better DFS, and the number of NLNs had a prognostic value in patients with different $\mathrm{pT}$ stages and in $\mathrm{pN}$ positive patients.

Because lymph node dissection includes positive lymph nodes, it is difficult to accurately estimate the appropriate number of lymph nodes to remove. Our results are consistent with those of other studies which have examined the prognostic value of the number of NLNs removed in breast cancer patients [12,13]. Karlsson et al. found that the number of NLNs removed was an independent factor affecting prognosis; patients with $\geq 10$ NLNs removed had a better prognoses than patients with $<10$ NLNs removed, which affected node positive patients but not node negative patients [12]. Kuru reported that patients with $>15$ NLNs removed had a better prognoses than those with fewer removed [13]. The mechanism underlying why the number of NLNs can be used to predict the survival of breast cancer patients is unclear. Insufficient lymph node dissection may result in inaccurate lymph node staging, and removing more lymph nodes makes for more accurate determination of the lymph node status. Therefore, studies have proposed the "stage migration" hypothesis that obtaining accurate information of lymph nodes and determining the lymph node stage by removing more lymph nodes to decrease the probability of error of nodal stage. Schaapveld et al. found that removing more lymph nodes resulted in a better survival, which supports the "stage migration" hypothesis [14].

At present, H\&E staining is a commonly used method for detecting positive lymph nodes. However, immunohistochemical methods can reveal occult lesions in lymph nodes $[15,16]$. It is obvious that an increased number of NLNs removed increases the potential for the identification of micrometastases. Because immunohistochemical methods were not used for examination of the lymph nodes in the present study, removing a higher number of NLNs might eliminate some potential remnant lesions, and this can explain the fact that patients with a higher number of NLNs removed had better DFS in the present study, which also supports the "stage migration" hypothesis. Moreover, it should be mentioned that the number of NLNs removed may be related to the host immune response against tumor cells, and the molecular biology of tumor cells $[17,18]$. A study examining colorectal cancers showed there was a significant correlation between lymphocyte response
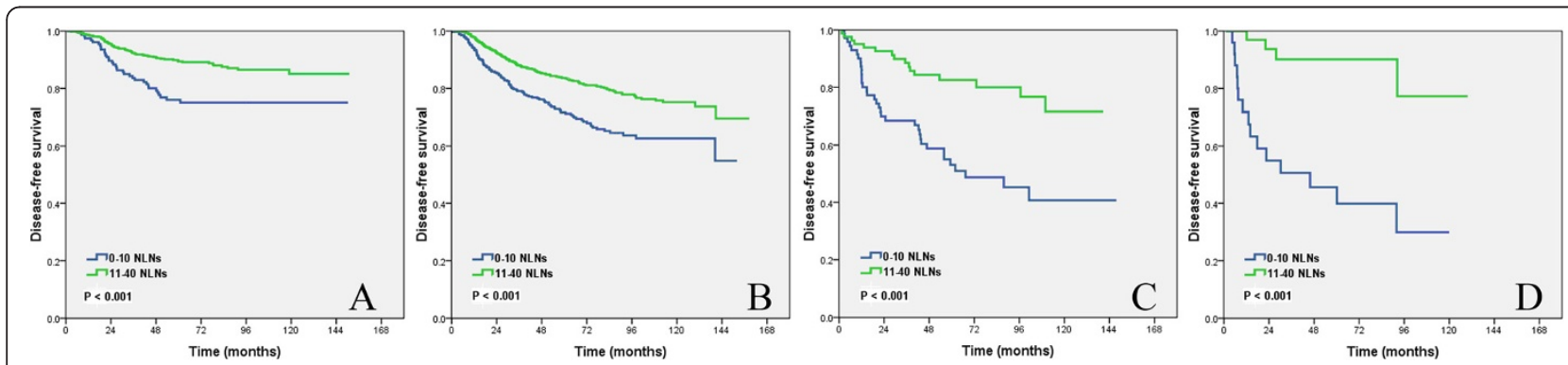

Figure 2 Impact of the number of negative lymph nodes on the disease-free survival of pT1 (A), pT2 (B), pT3 (C), and pT4 (D) patients. 

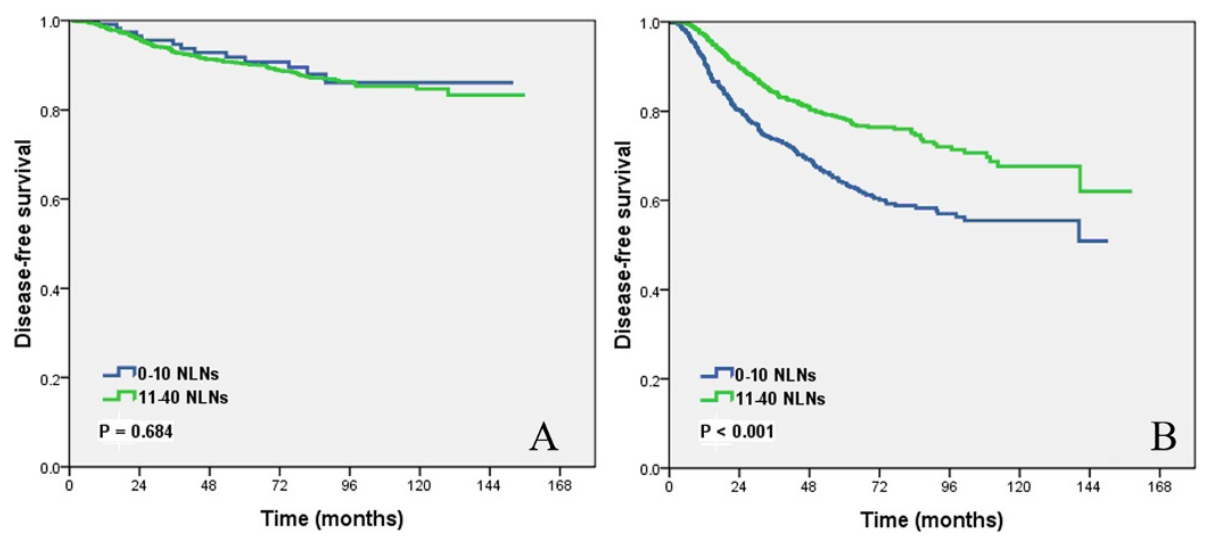

Figure 3 Impact of the number of negative lymph nodes on the disease-free survival of pNO patients (A) and pN positive patients (B).

against tumor cells and patients survival [19]. Hence, it is meaningful to further investigate the number of NLNs removed and the lymphocyte response against tumor cells for the individualized treatment of breast cancer.

The American College of Surgeons Oncology Group (ACOSOG) Z0011 trial showed that the local recurrence and survival rates of patients who underwent breastconserving surgery with negative sentinel lymph node or 1-2 positive sentinel lymph nodes were not affected by whether axillary lymph node dissection was performed or not, and thereby suggests that axillary lymph node dissection should not be carried out for these patients $[1,2]$. However, the report of the St-Gallen International Breast Cancer Conference in 2013 indicates that axillary lymph node dissection should be performed for patients who cannot receive radiotherapy or who have $\geq 3$ metastatic sentinel lymph nodes [20]. Z0011 trial participants received breast conserving surgery and radiotherapy, patients enrolled in the present study were treated with mastectomy and irradiation was performed based on the tumor stage and lymph node status.

In the present study, subgroup analysis showed that the number of NLNs removed only affected the DFS of node positive patients. Though it was reported that the number of removed lymph nodes might affect the local recurrence rate of node positive patients [21], we found that the number of NLNs removed did not affect the DFS of pN0 patients and thus consider that sentinel lymph node biopsy is sufficient for the determination of lymph node status in node-negative patients.

There are limitations of the present study that must be considered. First, the study was a single center retrospective study, and thus may not represent the majority of the population. However, the number of cases was large. Second, the optimal number of NLNs removed is not consistent with that of other studies. This may be related to differences in clinical data and surgical procedures, and prospective multicenter studies should be carried out to identify an exact value and a more appropriate cut-off number of the NLNs that should be removed.

\section{Conclusions}

In conclusion, our study indicates that the number of NLNs removed is an important factor affecting the DFS of breast cancer patients after mastectomy, and patients with a higher number of NLNs removed have a better prognosis. However, our result should be verified by further studies, and the related mechanism should be studied to provide a choice for the postoperative treatment of breast cancer.

\section{Abbreviations}

UICC/AJCC: Union for International cancer control/american joint committee on cancer; TNM: Tumor, node, metastasis; NLNs: Negative lymph nodes;

DFS: Disease-free survival; ER: Estrogen receptor; PR: Progesterone receptor Her2: Human epithelial growth factor receptor family 2; FISH: Fluorescent in situ hybridization; H\&E: Hematoxylin and eosin; HR: Hazard ratio; Cl: Confidence interval; ACOSOG: American College of Surgeons Oncology Group.

\section{Competing interests}

The authors declare that thay have no competing interests.

\section{Authors' contributions}

SGW, JYS, and JZ carried out the data collection and writing of the manuscript; SGW helped to conceive the study; SGW and ZYH contributed to the design of the study; QL and FYL helped to collect data, HXL and XXG participated in statistical analysis. All authors read and approved the final manuscript.

\section{Acknowledgments}

This work was supported by grants from the National Natural Science Foundation of China (No. 81402527), the Sci-Tech Office of Guangdong Province (No. 2013B021800157), Medical Scientific Research Foundation of Guangdong Province (A2010192), and the Education Scientific Research Project of Young Teachers in Fujian Province (No. JB13131).

\section{Author details}

'Xiamen Cancer Center, Department of Radiation Oncology, the First Affiliated Hospital of Xiamen University, Xiamen 361003, People's Republic of China. ${ }^{2}$ Department of Radiation Oncology, Sun Yat-sen University Cancer Center, State Key Laboratory of Oncology in South China, Collaborative Innovation Center of Cancer Medicine, Guangzhou 510060, People's Republic of China. ${ }^{3}$ Xiamen Cancer Center, Department of Obstetrics and Gynecology, 
the First Affiliated Hospital of Xiamen University, Xiamen 361003, People's Republic of China.

Received: 12 September 2014 Accepted: 29 January 2015 Published online: 07 February 2015

\section{References}

1. Sackey H, Magnuson A, Sandelin K, Liljegren G, Bergkvist L, Fülep Z, et al. Arm lymphoedema after axillary surgery in women with invasive breast cancer. Br J Surg. 2014;101(4):390-7.

2. De Gournay E, Guyomard A, Coutant C, Boulet S, Arveux P, Causeret S, et al. Impact of sentinel node biopsy on long-term quality of life in breast cancer patients. Br J Cancer. 2013;109(11):2783-91.

3. Giuliano AE, McCall L, Beitsch P, Whitworth PW, Blumencranz P, Leitch AM, et al. Locoregional recurrence after sentinel lymph node dissection with or without axillary dissection in patients with sentinel lymph node metastases: the American College of Surgeons Oncology Group Z0011 randomized trial. Ann Surg. 2010;252(3):426-32. discussion 432-433.

4. Giuliano AE, Hunt KK, Ballman KV, Beitsch PD, Whitworth PW, Blumencranz PW, et al. Axillary dissection vs no axillary dissection in women with invasive breast cancer and sentinel node metastasis: a randomized clinical trial. JAMA. 2011:305(6):569-75.

5. van der Wal BC, Butzelaar RM, van der Meij S, Boermeester MA. Axillary lymph node ratio and total number of removed lymph nodes: predictors of survival in stage I and II breast cancer. Eur J Surg Oncol. 2002;28(5):481-9.

6. Tausch C, Taucher S, Dubsky P, Seifert M, Reitsamer R, Kwasny W, et al. Prognostic value of number of removed lymph nodes, number of involved lymph nodes, and lymph node ratio in7502 breast cancer patients enrolled onto trials of the Austrian Breast and Colorectal Cancer Study Group (ABCSG). Ann Surg Oncol. 2012;19(6):1808-17.

7. Somner JE, Dixon JM, Thomas JS. Node retrieval in axillary lymph node dissections: recommendations for minimum numbers to be confident about node negative status. J Clin Pathol. 2004;57(8):845-8.

8. Zhu Z, Chen H, Yu W, Fu X, Xiang J, Li H, et al. Number of negative lymph nodes is associated with survival in thoracic esophageal squamous cell carcinoma patients undergoing three-Field lymphadenectomy. Ann Surg Oncol. 2014:21(9):2857-63.

9. Hsu PK, Huang CS, Wang BY, Wu YC, Chou TY, Hsu WH. The prognostic value of the number of negative lymph nodes in esophageal cancer patients after transthoracic resection. Ann Thorac Surg. 2013;96(3):995-1001.

10. Chen $Y$, Zhang $L$, Tian J, Ren $X$, Hao Q. Combining the negative lymph nodes count with the ratio of positive and removed lymph nodes can better predict the postoperative survival in cervical cancer patients. Cancer Cell Int. 2013;13(1):6

11. Ogino S, Nosho K, Irahara N, Shima K, Baba Y, Kirkner GJ, et al. Negative lymph node count is associated with survival of colorectal cancer patients, independent of tumoral molecular alterations and lymphocytic reaction. Am J Gastroenterol. 2010;105(2):420-33.

12. Karlsson P, Cole BF, Price KN, Coates AS, Castiglione-Gertsch M, Gusterson $B A$, et al. The role of the number of uninvolved lymph nodes in predicting locoregional recurrence in breast cancer. J Clin Oncol. 2007;25(15):2019-26.

13. Kuru B. Prognostic significance of total number of nodes removed, negative nodes removed, and ratio of positive nodes to removed nodes in node positive breast carcinoma. Eur J Surg Oncol. 2006;32(10):1082-8.

14. Schaapveld M, de Vries EG, van der Graaf WT, Otter R, de Vries J, Willemse $\mathrm{PH}$. The prognostic effect of the number of histologically examined axillary lymph nodes in breast cancer: stage migration or age association? Ann Surg Oncol. 2006;13(4):465-74.

15. Cote RJ, Peterson HF, Chaiwun B, Gelber RD, Goldhirsch A, CastiglioneGertsch $M$, et al. Role of immunohistochemical detection of lymph-node metastases in management of breast cancer. International Breast Cancer Study Group. Lancet. 1999;354(9182):896-900.

16. Inokuchi M, Ninomiya I, Tsugawa K, Terada I, Miwa K. Quantitative evaluation of metastases in axillary lymph nodes of breast cancer. $\mathrm{Br} J$ Cancer. 2003;89(9):1750-6.

17. Johnson PM, Porter GA, Ricciardi R, Baxter NN. Increasing negative lymph node count is independently associated with improved long-term survival in stage IIIB and IIIC colon cancer. J Clin Oncol. 2006;24(22):3570-5.

18. Pagès F, Berger A, Camus M, Sanchez-Cabo F, Costes A, Molidor R, et al. Effector memory T cells, early metastasis, and survival in colorectal cancer. N Engl J Med. 2005;353(25):2654-66.
19. Morris M, Platell C, lacopetta B. Tumor-infiltrating lymphocytes and perforation in colon cancer predict positive response to 5-fluorouracil chemotherapy. Clin Cancer Res. 2008;14(5):1413-7.

20. Goldhirsch A, Winer EP, Coates AS, Gelber RD, Piccart-Gebhart M, Thürlimann B, et al. Personalizing the treatment of women with early breast cancer: highlights of the St Gallen International Expert Consensus on the Primary Therapy of Early Breast Cancer 2013. Ann Oncol. 2013;24(9):2206-23.

21. Vujovic $O, Y u$ E, Cherian A, Dar AR, Stitt L, Perera F. The number of axillary nodes removed as a predictor of regional recurrence in node negative breast cancer. Radiother Oncol. 2009;91(1):38-41.

\section{Submit your next manuscript to BioMed Central and take full advantage of:}

- Convenient online submission

- Thorough peer review

- No space constraints or color figure charges

- Immediate publication on acceptance

- Inclusion in PubMed, CAS, Scopus and Google Scholar

- Research which is freely available for redistribution 